\title{
ÉTHIQUE DE LA RENCONTRE ET PENSÉE DÉCOLONIALE: ÉCRIVAINES AMÉRINDIENNES ET SUBSAHARIENNES
}

\author{
Carmen Mata Barreiro ${ }^{1}$
}

\begin{abstract}
Résumé: Dans ce début du XXIe siècle, des écrivaines originaires de territoires perçus comme périphériques, les univers amérindien et subsaharien, revendiquent non seulement l'espace de la parole mais surtout la décolonisation et le décentrement de la pensée. Faisant partie des « Ateliers de la pensée " en Afrique ( $c f$. Léonora Miano) ou participant à des expériences d'écritures croisées comme Aimititau! Parlons-nous! (2008) et Uashtessiu/Lumière d'automne (2010), dans lesquelles les écrivaines amérindiennes Joséphine Bacon et Rita Mestokosho se sont engagées, elles invitent nos sociétés à faire un travail de mémoire et à penser et construire ensemble le monde. Une démarche philosophique, politique et littéraire, nourrie par une éthique de la rencontre.
\end{abstract}

Mots-clés : amérindien, subsaharien, mémoire, réappropriation

\section{ÉTICA DEL ENCUENTRO Y PENSAMIENTO DESCOLONIAL: ESCRITORAS AMERINDIAS Y SUBSAHARIANAS}

\begin{abstract}
Resumen: En este inicio del siglo XXI, escritoras originarias de territorios percibidos como periféricos, los universos amerindio y subsahariano, reivindican no sólo el espacio de la palabra sino, sobre todo, la descolonización y la descentralización del pensamiento. Formando parte de los "Talleres del pensamiento" en Africa ( $c f r$. Léonora Miano) o participando en experiencias de intercambio de textos como Aimititau! Parlons-nous! (2008) y Uashtessiu/Lumière d'automne (2010), en las que las escritoras amerindias Joséphine Bacon y Rita Mestokosho se han implicado, invitan a nuestras sociedades a realizar un trabajo de memoria y a pensar y construir juntas el mundo. Un planteamiento filosófico, político y literario, nutrido por una ética del encuentro.
\end{abstract}

Conceptos clave: amerindio, subsahariano, memoria, reapropiación

\footnotetext{
1 Professora titular da Universidade Autônoma de Madri. Professora convidada na Universidade de Montreal. Membro do Conselho de Administração da Associação Internacional dos Estudos Quebequenses (AIEQ) e pesquisadora internacional do CIRM/CRIEM, Centro de pesquisas interdisciplinares sobre Montreal. Endereço eletrônico: carmatba@idecnet.com.
} 
Nous constatons que, à la maturité et à l'énergie de leurs voix répondent actuellement la maturité et l'énergie dans la visibilité éditoriale et dans la réception de la critique universitaire. Le chemin parcouru s'avère remarquable. Ainsi, en ce qui concerne la littérature amérindienne, Diane Boudreau, dans l'Introduction de son Histoire de la littérature amérindienne au Québec, écrivait en 1993 : «Il ne s'agit pas ici de reconnaître à tout prix la légitimité de cette littérature, [...] il est plus que temps que nous apprenions à connaître et à apprécier une littérature d'ici » ( BOUDREAU, 1993, p. 18), et manifestait «l'espoir que [la littérature amérindienne] soit un jour reconnue "( BOUDREAU, 1993, p. 18). En 2007, des écrivains de nation wendate, crie, mi'kmaq, métisse, nippissing, dénée, tépéhuane, kiowa et innue -dont Joséphine Bacon et Rita Mestokosho-, ont construit un projet d'écritures croisées avec des écrivains québécois qui a été dénommé Aimititau! Parlonsnous !, publié par Mémoire d'encrier en 2008 et dirigé par Laure Morali. Et cette expérience de dialogue et de rencontre a donné lieu à deux autres projets, basés sur le même principe et traduisant le même élan, à savoir Uashtessiu/Lumière d'automne, de Rita Mestokosho et Jean Désy (Mémoire d'encrier, 2010), et Nous sommes tous des sauvages, de Joséphine Bacon et José Acquelin (Mémoire d'encrier, 2011). Louis-Karl Picard-Sioui, écrivain, anthropologue et historien Wendat, dans la préface du livre Nous sommes des histoires. Réflexions sur la littérature autochtone, publié en 2018, mettait en relief « la richesse et la diversité des œuvres publiées au cours des quarante dernières années, avec une accélération notable de la production au XXIe siècle » (JEANNOTTE, LAMY et ST-AMAND, dirs, 2018, p. 6), et soulignait que cette littérature autochtone d'expression française "raconte notre humanité » : « il s'agit avant tout d'histoires humaines. Et donc, au potentiel universel » (ibid., 2018, p. 7). Et Joséphine Bacon, Natasha Kanapé Fontaine et Marie-Andrée Gill ont participé à la prise de parole, avec 38 autres poètes québécoises, "d'ici et d'ailleurs", dans Femmes rapaillées (DUVAL et YOUNSI, dirs, 2016), en vue d'honorer l'héritage et de s'engager dans l'avenir, et faire partie de ce qu'une autre poète invitée, Marie-Célie Agnant, a appelé un «chant ininterrompu»: "les femmes/ vois-tu/ sont un chant ininterrompu » (AGNANT, in DUVAL er YOUNSI, dirs, 2016, p. 5, 61). 
ÉTHIQUE DE LA RENCONTRE ET PENSÉE DÉCOLONIALE: ÉCRIVAINES AMÉRINDIENNES ET SUBSAHARIENNES

Il semblerait donc que la représentation de la littérature amérindienne comme une "littérature de survie " et de "résistance", proposée par Diane Boudreau (1993) et par Barbara Godard («resistance writing ", GODARD, 1990, p. 198-199) devrait être remise en question de nos jours. La volonté d'affirmation et l'éthique de la rencontre constituent des moteurs de la littérature amérindienne (GATTI, 2009 [2004]) ou autochtone (HAREL, 2017) du XXIe siècle, et cette volonté est exprimée dans l'épigraphe en tête du collectif Aimititau! Parlons-nous !, en empruntant les paroles de l'un des représentants majeurs de la pensée d'Afrique subsaharienne actuelle, Achille Mbembe :

Le Monde à venir sera fondé non seulement sur une éthique de la rencontre, mais également sur le partage des singularités [...] C'est à la faveur de ce partage et de cette communicabilité que nous produisons l'humanité. (MBEMBE, 2007, p. 10)

\section{La littérature africaine subsaharienne: Léonora Miano}

En ce qui concerne la littérature subsaharienne d'expression française, la génération que l'écrivain Abdourahman A. Waberi a appelée « les enfants de la postcolonie» (WABERI, 1998) et dont font partie Alain Mabanckou, Sami Tchak, Fatou Diome, Calixthe Beyala et Léonora Miano, a entrepris un travail d'élaboration d'une pensée critique qui remet en cause la prépondérance du discours occidental et tient à proposer des approches renouvelées et des concepts innovants qui aident à relire le monde et à produire de nouvelles intelligibilités sur les réalités et les devenirs de l'Afrique. La visibilité de la vitalité et la puissance de leur travail s'expose dans des publications récentes telles que Penser et écrire l'Afrique aujourd'hui (2017), dirigé par Alain Mabanckou, et Écrire l'Afrique-Monde(2017), fruit de la réflexion d'écrivains tels que Léonora Miano, Alain Mabanckou, Sami Tchak, en dialogue avec des intellectuels comme Achille Mbembe et Souleymane

\footnotetext{
${ }^{2}$ Maurizio Gatti, auteur de Littérature amérindienne du Québec. Écrits de langue française (2004, 2009), revendique le terme "amérindien » plutôt qu'autochtone " parce qu'il [lui] semble plus précis et sans équivoque " (GATTI, 2009 [2004], p. 13). Simon Harel choisit l'épithète " autochtone » dans sa récente étude Place aux littératures autochtones (2017), où il explique que «l'emploi des termes « autochtone » et « autochtonie » n'est pas anodin : il s'agit de signaler la présence d'un essentialisme nécessaire à la résistance néocoloniale, ici d'un point de vue culturel » (HAREL, 2017, p. 119, note 10).
} 
Bachir Diagne, dans le cadre des Ateliers de la pensée tenus à Dakar et à SaintLouis-du-Sénégal en 2016.

À l'intérieur de la génération des "enfants de la postcolonie», Léonora Miano propose une littérature qui -disait-elle en 2012- "réfléchit autant qu'elle raconte » (MIANO, 2012, p. 7), en romancière et en essayiste. Sa posture d'affirmation et de dialogue détermine le choix et la configuration des genres qu'elle investit, sa posture "diasporale " (MALELA, 2011, p. 163), -se définissant comme une auteure "d'expression française, mais de culture africaine et afro-américaine " (MIANO, Afropean soul et autres nouvelles entretien-, 2008, p. 94)-, ainsi que son identification avec une "identité frontalière " associée à une perception de la frontière non pas comme un lieu de rupture mais comme un espace de rencontre et "d'accolement permanent » (MIANO, 2012, p. 25).

Son œuvre est constituée de sept romans dont le premier, L'intérieur de la nuit, a été publié en 2005, et de trois recueils de textes, Écrits pour la parole (2012), conçus pour le théâtre, et Habiter la frontière (2012) et L'impératif transgressif. Communications - Réflexions (2016a), des essais où Miano approfondit les problématiques de l'identité, de l'histoire et de la mémoire d'Afrique subsaharienne et des afrodescendants, en intellectualisant sa perspective critique.

En effet, l'un des traits caractéristiques de l'œuvre de Léonora Miano est le dialogue voire l'alliage entre création et théorie et entre écriture réflexive et dimension politique. Ainsi, dans le roman Les aubes écarlates. "Sankofa cry» (2009), l'un de ses romans du triptyque sur l'Afrique et la traite négrière, c'est le péritexte qui accueille la réflexion de Nathalie Etoke sur l'élaboration d'une conscience diasporique, qu'elle nomme « Melancholia Africana », " concept extensible qui examine comment les Noirs gèrent la perte, le deuil et la survie » (ETOKE in Miano, 2009, p. 270-271); et ce concept nourrit le premier volume, Melancholy (2016b), de son roman choral Crépuscule du tourment (MIANO, 2016-2017), auquel répond, en 2017, le second volume, Heritage. Et dans L'impératif transgressif. Communications Réflexions (MIANO, 2016a), l'écrivaine cherche à contribuer à la " réhabilitation de la conscience de soi au sud du Sahara » (MIANO, 2016a, p. 7) tout en proposant un apport épistémologique. 


\section{Imaginaire décolonial, récit sur l'histoire et discours : approche comparative}

Dans les deux univers littéraires que nous abordons ici, les écrivaines étudiées entreprennent un travail d'écoute et un "travail de mémoire »(RICOEUR, 2000), ainsi qu'un travail de déconstruction de discours et de concepts.

Travail de mémoire et "écriture du traumatisme " (ST-AMAND, 2010)

"[L]'effacement de la mémoire e[st] un des premiers gestes de la domination » (MIANO, 2017, p. 112), et le travail de mémoire tarde à se mettre en place sur le continent africain. Léonora Miano propose de "libérer la parole » et de " briser ses propres silences pour se dire pleinement " (MIANO, L'impératif transgressif, 2016a, p. 63) :

Peu importe, au fond, que l'Occident ne soit pas pressé de modifier son regard sur nous, pour nous étreindre fraternellement. Il nous appartient d'effectuer ce mouvement en direction de nous-mêmes. Il nous appartient de retourner à nous-mêmes [...] afin que nous ne soyons, à nos propres yeux, que l'image façonnée par d'autres. C'est à nous qu'incombe la tâche de dire, non pas comment nous sommes, mais qui. C'est à ce prix qu'auront lieu les nécessaires échanges qui manquent encore. (MIANO, 2016a, p. 62-63).

La Déportation transatlantique des Subsahariens lui semble être « le grand impensé des littératures subsahariennes " (MIANO, 2016a, p. 63). Une violence et une blessure qui touche les populations descendantes des Subsahariens déportés aux Amériques et aussi une violence sur le sol ancestral, une violence qui « endeuille et mutile » (MIANO, 2017, p. 111) : « nous sommes certes les descendants de nos ancêtres, mais nous sommes surtout le fruit des violences qui leur furent infligées " (MIANO, 2017, p. 111). Les récits de la capture, la déportation, la plantation, la colonie et l'exclusion sociale, qui sont l'héritage reçu par les Afrodescendants et les Afropéens ${ }^{3}$, s'avèrent

${ }^{3}$ Afropéens : contraction d'Afroeuropéens. 
incontournables en vue d'entreprendre le chemin de « la fraternité » (MIANO, 2016a, p. 87).

À cela s'ajoute une autre violence, celle inhérente à la colonisation par l'Europe de l'Ouest. Une colonisation de l'imaginaire poussée par une volonté d'assimilation d'une Europe "conquérante et prédatrice " (MIANO, 2017, p. 102), qui tient à imposer des éléments inconciliables avec les systèmes de pensée locaux et qui sont susceptibles de dévitaliser les sociétés qu'ils inoculent, sur le plan collectif et sur le plan intime. Ainsi, dans le premier volume du roman Crépuscule du tourment (2016-2017), Melancholy (MIANO, 2016b), où quatre femmes s'adressent au même homme, la première femme, " Madame ", sa mère, en évoquant les «blessures souterraines » (2016b, p. 9), la "fêlure » (2016b, p. 10) et la "faille» (2016b, p. 10), se penche sur la colonisation religieuse, sur l'assujettissement à des normes qui emprisonnaient les corps des femmes et sur la soumission à des récits qui leur étaient incompréhensibles:

Nos aînées nous envoyèrent à l'église, au temple. Le corps contraint par des tenues ajustées à la taille. Les seins emprisonnés dans les armatures métalliques du soutiengorge. [...] On saluait la Vierge, l'Immaculée, qu'une prophétie avait rendue grosse. [...] Je me suis longtemps demandé ce qu'il y avait à tirer d'une telle figure, pour une femme vivant sur cette terre. Quels étaient les mérites de cette Vierge, de façon concrète. Sainte Marie, Mère de Dieu... Élue entre toutes pour enfanter un illuminé, [...] Que ne dirait-on pas, de par le monde, si nous avions été les auteurs de semblables billevesées. (MIANO, 2016b, p. 14-15)

Chez les écrivaines amérindiennes, l'écriture de la rupture et de la perte exprime la violence inhérente à la colonisation et à la dépossession, qui touche la terre, l'imaginaire, l'identité et le corps des femmes.

Certaines écrivaines expriment la colère et la résistance aux différentes manifestations de violence subie. Le récit de l'Innue An Antane Kapesh, Je suis une maudite sauvagesse/Eukuan nin matshimanitu innuiskueu, a ouvert la voie en 1976. Il s'agit du récit de son expérience des relations avec le "Blanc» sur son territoire, le Nitassinan, un livre de 
ÉTHIQUE DE LA RENCONTRE ET PENSÉE DÉCOLONIALE: ÉCRIVAINES AMÉRINDIENNES ET SUBSAHARIENNES

mémoire pour les Innues et les Innus, et de dialogue avec les allochtones. La violence de son écriture traduit sa posture politique de résistance à la colonisation ainsi que son amour et sa fierté du mode de vie traditionnel, l'innu aitun. Elle dénonce et analyse la violence provoquée par le «Blanc » comme catégorie politique d'habitation (MAILHOT, 2017, p. 30) : la violence déterminée par son rapport au territoire qui entraîne sa destruction, par sa mise en récit de la colonisation et par son regard sur les Amérindiens qui exprime le manque de considération à leur égard, perçus comme « des êtres non civilisés » (ANTANE KAPESH, 1976, p. 155).

$\mathrm{Au}$ XXIe siècle, la blessure s'exprime particulièrement dans la poésie. Dans le poème « Je suis la Femme Rapaillée », intégré dans le collectif Femmes rapaillées (2016), Natasha Kanapé Fontaine, poète, slameuse, peintre et comédienne innue, évoque le rapport au territoire et se focalise sur la violence à l'encontre des femmes autochtones, violence systémique qui découle des traumatismes issus de la colonisation ${ }^{4}$ :

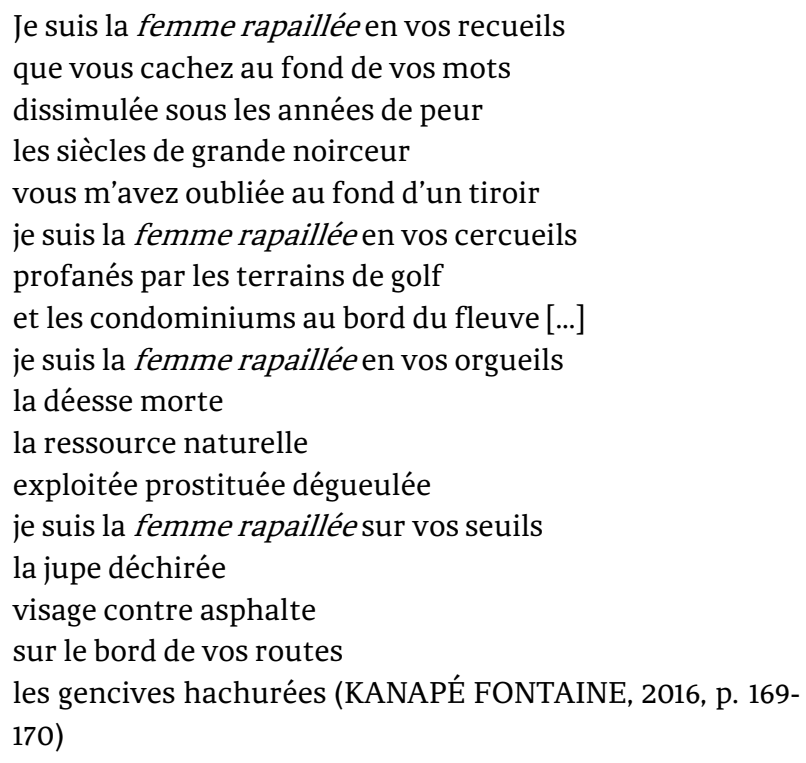

\footnotetext{
${ }^{4}$ En 2014 et 2015, deux ouvrages visent à mettre fin au silence sur le féminicide touchant les filles et les femmes autochtones au Canada et au Québec: Emmanuelle Walter, Soeurs volées: enquête sur un féminicide au Canada, Montréal, Lux Éditeur, 2014, et le rapport de Femmes Autochtones du Québec (FAQ), Nânîawig Mâmawe Nînawind. Debout et solidaires. Femmes autochtones disparues ou assassinées au Québec, Kahnawake, Femmes autochtones du Québec, 2015.
} 
Joséphine Bacon, Innue de Betsiamites, fait face au "silence » (BACON, 2009, p. 56) et a recours à la poésie pour exprimer sa douleur et sa lutte "dans une colère tranquille" (BACON, 2018, p. 44). Dans son premier recueil de poésie Bâtons à message. Tshissinuatshitakana (2009), elle évoque la rupture dans la transmission des valeurs culturelles et de l'identité, subie dans son enfance et adolescence au pensionnat, ce que le rapport final de la Commission de vérité et réconciliation du Canada dénomme "génocide culturel $^{5} »$ :

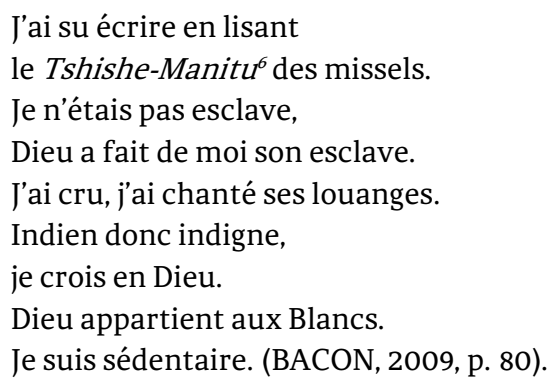

\section{De la blessure aux " cicatrices»}

Léonora Miano rejoint Joséphine Bacon dans l'idée de la cohabitation entre la prise de conscience de se savoir issue de profondes blessures et la représentation des " cicatrices " comme une voie de résilience. Bacon évoque les « cicatrices qui pardonnent » (BACON, 2018, p. 36) dans son recueil Uiesh. Quelque part (2018). Léonora Miano écrit que « c'est sur le sol ancestral qu'il faut se confronter aux conséquences de la violence, apprendre à en arborer avec grâce les cicatrices » (MIANO, 2017, p. 107) pour réussir à construire « une africanitéguérie de ses meurtrissures » (MIANO, 2017, p. 109).

\section{Décoloniser le langage}

Léonora Miano et Joséphine Bacon abordent une autre manifestation de dépossession, à savoir les désignations traduisant le discours de l'autre sur soi : Afrique et Africains, Noirs et Sauvages. Dans un article faisant partie du collectif Écrire l'Afrique-Monde (2017), Miano se

\footnotetext{
${ }^{5}$ Commission de vérité et réconciliation du Canada, Honorer la vérité, réconcilier pour l'avenir. Sommaire du rapport final de la Commission de vérité et réconciliation du Canada, 2015, p. 1.

${ }^{6}$ Tshishe-Manitu: Dieu ou le Grand esprit, "Lexique”, Bacon, 2009, p. 139.
} 
ÉTHIQUE DE LA RENCONTRE ET PENSÉE DÉCOLONIALE: ÉCRIVAINES AMÉRINDIENNES ET SUBSAHARIENNES

penche sur le nom Afrique "inconnu de nos ancêtres précoloniaux, et cependant devenu nôtre » (MIANO, 2017, p. 103). Plutôt que de l'extirper et de le remplacer, Miano propose d'en interroger les significations pour les Africains, "pour nous-mêmes" (MIANO, 2017, p. 106). Afrique devrait être perçue non pas comme une simple désignation mais comme une "narration, une histoire déjà multiséculaire dont il convient aujourd'hui de forger le sens afin de la posséder pleinement »(MIANO, 2017, p. 106).

La volonté de dépasser la blessure rejoint ainsi, sur le plan du langage, la volonté de dépasser le stigmate. Il ne s'agit pas de nier ou d'oublier le fait que "[1]es langues, qui sont des systèmes de pensée, ont été des instruments d'assujettissement, les outils d'une pénétration par effraction au cœur de visions du monde qu'elles ont contribué à brouiller » (MIANO, 2017, p. 106). Mais l'écrivaine envisage " une pensée d'Afrique renouvelée » (MIANO, 2017, p. 109), « un projet de civilisation original et souverain » (MIANO, 2017, p. 113), qui se fonderait sur les "universaux subsahariens [...] et diasporiques " (MIANO, 2017, p. 109, 114) dont la melancholia africana que Nathalie Etoke présente comme une aptitude à « résister au déclin, à révérer la vie contre tout ce qui s'évertue à la profaner » (ETOKE, 2010, p. 28). D'après Miano, la melancholia africana « émane des forces spirituelles de peuples auxquels elle permet de se hisser au-dessus de la douleur » (MIANO, 2017, p. 114).

En ce qui concerne l'épithète "Noirs", c'est le personnage d'Amandla, du roman choral Crépuscule du tourment. I. Melancholy, qui y réfléchit particulièrement. Amandla, afrodescendante, originaire de « [c]e pas coincé entre Brésil et Surinam. [...] La terre de déportation » (MIANO, 2016b, p. 100,99 ), refuse « le nom racial de Noirs pour nous désigner » (MIANO, 2016b, p. 82), qui ne fit jamais référence à leurs « trente-six carnations " (MIANO, 2016b, p. 83). Sa mère, Aligossi, lui avait appris à « récuser les appellations par lesquelles notre identité fut bafouée " (MIANO, 2016b, p. 82), et elle estime que ce nom racial fait partie du processus de dépossession et de réification entrepris par les envahisseurs du temps jadis: «[j]ustifier la dispersion transatlantique. Faire de nous des biens meubles que l'on achèterait à

\footnotetext{
${ }^{7}$ Nathalie Etoke, Melancholia Africana, L'indispensable dépassement de la condition noire, Paris, Éditions du Cygne, 2010.
} 
tempérament. Des bêtes que l'on marquerait au fer rouge avant de les baptiser selon le rite chrétien » (MIANO, 2016b, p. 82-83).

Dans le livre Nous sommes tous des sauvages (2011), présenté comme " chronique », la poète innue Joséphine Bacon est accompagnée par le poète québécois José Acquelin dans son approche de cette épithète qui traduit le mépris accumulé par l'Europe colonisatrice. Dès leur poème "Avant nous ", qu'ils cosignent en guise d'introduction, nous constatons la volonté d'affranchir ce mot de "la grandiose illusion civilisatrice " (cf. HAMELIN, « Postface », p. 67) et de le ré-enraciner, en ayant recours à la poésie:

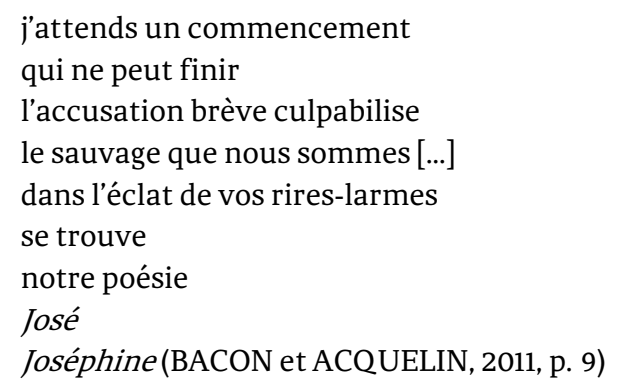

Joséphine Bacon dénonce le refus de regarder et d'écouter l'autre autochtone, qui est sous-jacent à cette désignation, et revendique le droit à une identité et à un récit:

je rêve d'un seul récit qui dicterait sans faute toute une vie vécue tu ne me regardes pas tu ne me vois pas tu ne m'entends pas tu ne m'écoutes pas tu ne me parles pas tu es ici en conquérant de ma Terre tu m'emprisonnes dans ma Terre tu me prives de mon identité tu me prives de mon territoire [...] qui suis-je ? Joséphine, (BACON et ACQUELIN, 2011, p. 10) 
À la fin de ce chemin de réflexion poétique, Joséphine Bacon dénomme son univers et met en relief son appartenance au genre humain, à l'humanité:

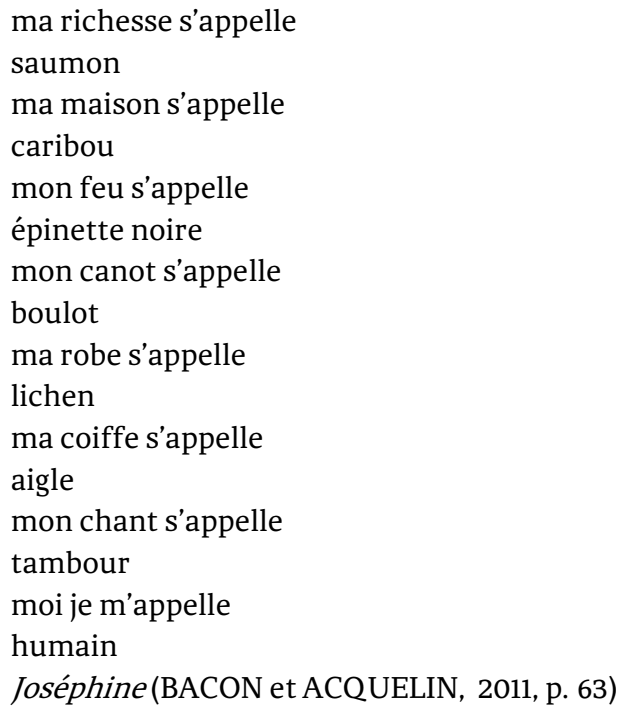

Ce poème, le dernier que Joséphine Bacon signe en solo dans Nous sommes tous des sauvages, est une profession de foi qui revendique son appartenance à l'humanité et aussi à un territoire innu, le Nutshimit, l'intérieur des terres, terres ancestrales, espace géosymbolique chargé de significations.

\section{La réappropriation culturelle : langue, langages et territoire}

Dans l'œuvre de Joséphine Bacon, le Nutshimit est perçu comme un territoire-sanctuaire, un espace de communion avec un ensemble de signes et de valeurs, un espace où elle a recours au langage des " bâtons à message ${ }^{8}$ " comme geste et trace visuelle de transmission et de partage:

Quelque part

Dans le Nutshimit

Je suis chez moi

\footnotetext{
${ }^{8}$ Son premier recueil de poèmes a comme titre Bâtons à message. Tshissinuatshitakana, Montréal, Mémoire d'encrier, 2009.
} 


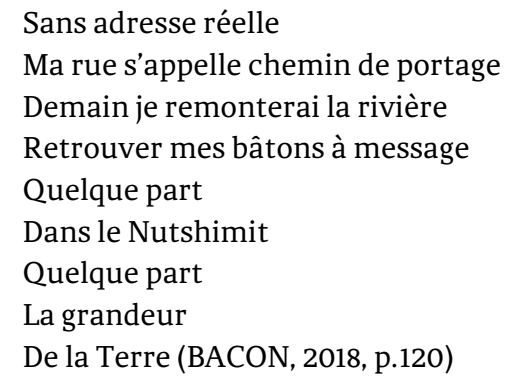

De même que dans le roman Kuessipan. À toi (2011), de la jeune écrivaine innue Naomi Fontaine, le je énonciateur du recueil Uiesh. Quelque part de Bacon part au Nutshimit pour « entendre ce que la terre de ses ancêtres a à lui dire " (FONTAINE, 2011, p. 66). Le territoire devient ainsi un " lieu de mémoire » (NORA, 1984-1992) où s'enracine la pensée innue, et où se met en scène sa communion avec la nature et avec ses mythes:

\author{
J'ai souvenir de Shuaushemiss \\ Grand-père chasseur \\ Je le revois avec son tambour \\ Il chante une femme aux cheveux blancs \\ Son chant pousse à la danse (BACON, 2018, p. 50)
}

La poésie devient ainsi, dans l'œuvre de Bacon, la voie de son réenracinement, de la lutte contre la dépossession. Les poèmes, en français et en innu-aimun, lui permettent de rejoindre l'oralité ( La tradition orale rassure mes peurs/ de blanc-mémoire", Bacon, Un thé dans la toundra. Nipishapui nete mushuat, 2013, p. 56) et "L'âme de la langue/ De mes ancêtres », qu'elle dépeint comme " un son/ Né du cri de la rivière » (Bacon in Aimititau ! Parlons-nous !, dir. par Laure Morali, 2017, p. 304).

L'évocation des ancêtres - « les ancêtres sont en nous, [...] ils nous accompagnent où que nous soyons » (MIANO, 2017, p. 112)- et la revendication d'une "pensée afrophonique»(MIANO, 2016a, p. 69), qui fait converger l'expression en langues subsahariennes et " des contenus, des ressources où puiser pour forger de nouvelles subjectivations» (MIANO, 2016a, p. 99), sont perçus et conçus par Miano comme un patrimoine et une "parole transnationale et culturellement transversale [...] pour penser [...] ce que doit être l'apport du continent à la marche du monde » (MIANO, 2016a, p. 99-100). 
L' " afrophonie », telle qu'elle l'envisage, est « le discours qui s'énonce entre Subsahariens" ainsi que " la mise en dialogue, par les Subsahariens et les Afrodescendants de leurs expériences »(MIANO, 2016a, p. 99-100).

La dimension éducative de l'afrophonie et l'importance de la transmission aux jeunes gens du patrimoine touchant les cultures et les sensibilités associées aux langues subsahariennes et afrodescendantes sont évoquées dans des essais de Miano tels que L'impératif transgressif, et incarnées dans des personnages féminins du roman Crépuscule du tourment. I. Melancholy. Ainsi, Amandla, consciente de ce qu'on « nous a dérobé. [...] Des morceaux de notre âme" (MIANO, 2016b, p. 89), réfléchit sur "[c]omment redevenir nous-mêmes en totalité " (MIANO, 2016b, p. 89), et se consacre à " restaurer notre vision du monde [...] [et à] [n]ous restituer l'usage de la parole " (MIANO, 2016b, p. 106). Ayant quitté le Nord et s'étant installée en Afrique subsaharienne, sur «la Terre première » (MIANO, 2016b, p. 84), elle transmet " notre tradition intellectuelle et spirituelle » en vue d' "inventer notre modernité » (MIANO, 2016b, p. 106). Ce sont surtout les enfants qui sont les destinataires de cette transmission. Amandla partage avec eux la mémoire des "résistants du Continent » (MIANO, 2016b, p. 106), afin qu'ils prennent conscience du fait que les peuples africains ont eu du courage face aux envahisseurs, mais que "[n]ous n'étions pas assez sauvages pour affronter leur barbarie " (MIANO, 2016b, p. 106). Elle leur dévoile des périodes douloureuses de leur Histoire: "Les travaux forcés. Les déplacements de populations. Le code de l'indigénat. La ségrégation raciale. Le génocide des Hereros " (MIANO, 2016b, p. 107). Et face à leur colère, elle les calme en leur expliquant que, plutôt que de "haïr» (MIANO, 2016b, p. 107), il serait plus important de travailler pour " rebâtir », pour " nous réparer [...] sans haine » (MIANO, 2016b, p. 107).

\section{En guise de conclusion : reconnaître l'autre, construire ensemble}

Léonora Miano et Joséphine Bacon -de même que Rita Mestokosho, Naomi Fontaine et Natasha Kanapé Fontaine- invitent les lecteurs autochtones et allochtones, africains, afrodescendants et afropéens, à se reconnaître eux-mêmes et les uns les autres; elles luttent pour que leurs 
peuples soient respectés dans leur humanité, leur complexité et la force de leur tradition et de leur imaginaire.

«Se libérer de la domination -écrivait Miano dans L'ímpératif transgressif-, c'est aussi refuser de lui emprunter ses méthodes : la négation de l'autre, son enfermement dans une altérité négative. S'affranchir de la domination, c'est s'attacher à diffuser d'autres valeurs, absolument » (MIANO, 2016a, p. 101)

Leur posture d'affirmation et leur volonté de rencontre et de dialogue cherchent à replacer leurs peuples dans la conscience humaine globale. Elles participent à une prise de conscience de l'urgence du décentrement du regard et de l'affranchissement de l'européocentrisme en rejoignant ainsi la lutte d'historiens de différentes cultures historiographiques tels que Patrick Boucheron, co-auteur de Pour une histoire-monde (2013), Jack Goody, auteur de Le vol de l'histoire. Comment l'Europe a imposé le récit de son passé au reste du monde (2010 [2006], The Theft of History) et Josep Fontana, auteur de Europa ante el espejo (Barcelone, 2000 [1994]).

Elles rejoignent aussi la réflexion d'une écrivaine et sociologue mohawk, Patricia A. Monture, qui, dans un article intitulé "Les mots des femmes [Women's Words]. Pouvoir, identité et souveraineté indigène", souligne que les récits de son peuple, les Haudenosaunee, enseignent ce que sont "l'identité et la responsabilité » (MONTURE, 2017 [2008], p. 15), et que " écrire, pour plusieurs auteurs ou auteures indigènes, est souvent un geste qui qualifie à la fois le pouvoir et l'exclusion, [...] [que] ce geste d'affirmation est souvent conçu comme le fait d'une résistance, alors que, bien souvent, c'est un geste de (ré)appropriation » (MONTURE, 2017 [2008], p. 20).

Monture s'y penche sur les différences entre les écrits européens, africains et ceux des Premières Nations, sur le plan du lieu de la connaissance et de l'énonciation, et a recours à l'analyse de Gloria Ladson-Billings (2000):

Quand René Descartes a affirmé que son geste de pensée l'amenait à l'existence, il articulait du même coup un $a$ priori fondamental de la vision du monde et de l'épistémologie européennes (et euro-américaines), à savoir que la conscience de l'individu est la source du 
savoir et de l'existence. On peut lui opposer ce dicton africain, " Ubuntu », que l'on peut traduire par « je suis parce que nous sommes », qui affirme que l'expérience individuelle (et la connaissance) dépend des relations que nous entretenons avec les autres. (LADSON-BILLINGS, 2000, p. 257).

L'étude de l'œuvre des écrivaines amérindiennes et subsaharienne que nous avons abordée ici, dans une approche transdisciplinaire où le genre et la dimension politique constituent des éléments importants de l'analyse, fait apparaître des voix puissantes qui exposent des problématiques telles que la sororité et l'homosexualité féminine en Afrique, la violence issue de la colonisation, le rapport au territoire, l'imaginaire politique, le racisme, et le pouvoir des mots.

Ces écritures au féminin s'avèrent incontournables en vue de mieux connaître les blessures du passé et leurs traces et afin de construire l'avenir sur les piliers de la lucidité, la vérité et l'éthique.

\section{Références}

ANTANE KAPESH, An (1976), Eukuan nin matshimanitu innu-iskueu/Je suis une maudite sauvagesse, Montréal, Leméac, coll. « Dossiers ».

BACON, Joséphine (2009), Bâtons à message. Tshissinuatshitakana, Montréal, Mémoire d'encrier.

BACON, Joséphine (2013), Un thé dans la toundra. Nipishapui nete mushuat, Montréal, Mémoire d'encrier.

BACON, Joséphine (2018), Uiesh. Quelque part, Montréal, Mémoire d'encrier.

BACON, Joséphine et ACQUELIN, José (2011), Nous sommes tous des sauvages. Chronique, Montréal, Mémoire d'encrier.

BOUCHERON, Patrick et DELALANDE, Nicolas (2013), Pour une histoiremonde, Paris, Presses Universitaires de France, coll. « La Vie des Idées ».

BOUDREAU, Diane (1993), Histoire de la littérature amérindienne au Québec: oralité et écriture, Montréal, L’Hexagone, coll. « Essai ».

DUVAL, Isabelle et YOUNSI, Ouanessa (dir), Femmes rapaillées, Montréal, Mémoire d'encrier. 
ETOKE, Nathalie (2010), Melancholia Africana. L'indispensable dépassement de la condition noire, Paris, Éditions du Cygne.

FONTAINE, Naomi (2011), Kuessipan. À toi, roman, Montréal, Mémoire d'encrier.

FONTANA, Josep (2000 [1994]), Europa ante el espejo, Barcelona, Ed. Crítica.

GATTI, Maurizio (2009 [2004]), Littérature amérindienne du Québec. Écrits de langue française, Montréal, Bibliothèque québécoise.

GODARD, Barbara (1990), "The Politics of Representation: Some Native Canadian Women Writers", in NEW, W.H. (dir), Native Writers, Canadian Writing, Vancouver, University of British Columbia Press, p. 183-225.

GOODY, Jack (2010 [2006]), Le vol de l'histoire. Comment l'Europe a imposé le récit de son passé au reste du monde [The Theft of History], Paris, Gallimard, coll. « Folio Histoire ».

HAMELIN, Louis (2011), "Postface. Développement sauvage", in BACON, Joséphine et ACQUELIN, José, Nous sommes tous des sauvages, Montréal, Mémoire d'encrier, p. 67-70.

HAREL, Simon (2017), Place aux littératures autochtones, Montréal, Mémoire d'encrier.

JEANNOTTE, Marie-Hélène, LAMY, Jonathan et ST-AMAND, Isabelle (dirs) (2018), Nous sommes des histoires. Réflexions sur la littérature autochtone, Montréal, Mémoire d'encrier.

KANAPÉ FONTAINE, Natasha (2016), "Je suis la Femme Rapaillée», in DUVAL, Isabelle et YOUNSI, Ouanessa (dirs), Femmes rapaillées, Montréal, Mémoire d'encrier, p. 166-171.

LADSON-BILLINGS, Gloria (2000), « Racialized Discourses and Ethnic Epistemologies », in DENZIN, Norman K. et LINCOLN, Yvonna S. (dirs), Handbook of Qualitative Research, Thousand Oaks, Sage Publications.

MABANCKOU, Alain (dir) (2017), Penser et écrire l'Afrique aujourd'hui, Paris, Seuil.

MAILHOT, Amélie-Anne (2017), « La perspective de l'habitation politique dans Je suis une maudite sauvagesse/Eukuan nin matshimanitu innu-iskueu d'An Antane Kapesh », Recherches féministes, vol. 30, no 1, p. 29-45. 
ÉTHIQUE DE LA RENCONTRE ET PENSÉE DÉCOLONIALE:

ÉCRIVAINES AMÉRINDIENNES ET SUBSAHARIENNES

MALELA, Buata B. (2011), " Jeu littéraire et transformation du sujet diasporal: La postmodernité de Léonora Miano ", Revue de l'Université de Moncton, vol. 42, no 1-2, p. 153-174.

MBEMBE, Achille (2007), "Francophonie et politique du Monde », p. 1-11, www.congopage.com

MBEMBE, Achille et SARR, Felwine (dir) (2017), Écrire l'Afrique-Monde, Dakar, Philippe Rey/Jimsaan.

MESTOKOSHO, Rita et DÉSY, Jean (2010), Uashtessiu/Lumière d'automne, Montréal, Mémoire d'encrier.

MIANO, Léonora (2008), Afropean soul et autres nouvelles, Flammarion, Coll. "Étonnants Classiques ».

MIANO, Léonora (2009), Les aubes écarlates. "Sankofa cry», Paris, Éd. Plon.

MIANO, Léonora (2012), Habiter la frontière. Conférences, Paris, L'Arche Éditeur.

MIANO, Léonora (2016a), L'impératif transgressif. CommunicationsRéflexions, Paris, L’Arche Éditeur.

MIANO, Léonora (2016b), Crépuscule du tourment. I. Melancholy, Paris, Bernard Grasset.

MIANO, Léonora (2017), " De quoi Afrique est-il le nom », in MBEMBE, Achille et SARR, Felwine (dir), Écrire l'Afrique-Monde, Dakar, Philippe Rey/Jimsaan, p. 99-115.

MONTURE, Patricia (2017 [2008], "Les mots des femmes [Women's Words]: Pouvoir, identité et souveraineté indigène ", Recherches féministes, vol. 30, no 1 , p. 15-27.

MORALI, Laure (dir), Aimititau ! Parlons-nous !, Montréal, Mémoire d'encrier.

NORA, Pierre (1984-1992), Lieux de mémoire, Paris, Gallimard.

RICOEUR, Paul (2000), La mémoire, l'histoire, l'oubli, Paris, Éditions du Seuil.

ST-AMAND, Isabelle (2010), « Discours critiques pour l'étude de la littérature autochtone dans l'espace francophone du Québec », Studies in Canadian Literature/ Études en littérature canadienne, Vol. 35, nำ, p. 1-10.

WABERI, Abdourahman (2017), « "Les enfants de la postcolonie" précédé d'une note liminaire", in MABANCKOU, Alain, Penser et écrire l'Afrique aujourd'hui, Paris, Seuil, p. 148-161. 
Recebido em 20 de junho de 2019.

Aceito em 25 de julho de 2019. 